\title{
Finslerian Quantum Field Theory
}

\author{
Howard E. Brandt \\ U.S. Army Research Laboratory, Adelphi, Maryland, U.S. \\ hbrandt@arl.army.mil
}

November 14, 2018

\begin{abstract}
Finsler geometry motivates a generalization of the Riemannian structure of spacetime to include dependence of the spacetime metric and associated invariant tensor fields on the four-velocity coordinates as well as the spacetime coordinates of the observer. It is then useful to consider the tangent bundle of spacetime with spacetime in the base manifold and four-velocity space in the fiber. A physical basis for the differential geometric structure of the spacetime tangent bundle is provided by the universal upper limit on proper acceleration relative to the vacuum. It is then natural to consider a quantum field having a vanishing eigenvalue when acted on by the Laplace-Beltrami operator of the spacetime tangent bundle. On this basis a quantum field theory can be constructed having a built-in intrinsic regularization at the Planck scale, and finite vacuum energy density.
\end{abstract}

\section{FINSLERIAN FIELDS}

A physical Finslerian field $F(x . v)$ is one that depends not only on the observer's spacetime coordinates,

$$
x \equiv\left\{x^{\mu}\right\}=\left\{x^{0}, x^{1}, x^{2}, x^{3}\right\},
$$

but also on the observer's four-velocity coordinates,

$$
v \equiv\left\{v^{\mu}\right\}=\left\{d x^{\mu} / d s\right\}=\left\{v^{0}, v^{1}, v^{2}, v^{3}\right\},
$$

where $d s$ is the infinitesimal interval along the worldline of the observer [1]-[3]. The four-velocity coordinates play here the role of the tangent space coordinates of Finsler geometry. It can be argued that the spacetime-metric field $g_{\mu \nu}$ itself must in general depend not only on where it is observed in spacetime, but also on the four-velocity of the 'observer', namely it is a Finslerian field [1]-[3]:

$$
g_{\mu \nu}=g_{\mu \nu}(x, v) .
$$


(The reader may prefer to replace the word 'observer' by 'measuring device', 'object acted upon by the field', or 'some other field interacting locally with the field'.) The spacetime metric in a canonical Finsler spacetime is not only Finslerian, but also satisfies special homogeneity conditions involving the dependence of the metric on the tangent space coordinates, $v$. In particular, one has [4], [5]

$$
d s=L(x, d x),
$$

where $L$ is the fundamental Finsler function, and

$$
L(x, a d x)=a L(x, d x),
$$

from which it follows that

$$
\begin{gathered}
g_{\mu \nu}=\frac{1}{2} \frac{\partial^{2}}{\partial v^{\mu} \partial v^{\nu}} L^{2}(x, v), \\
L^{2}(x, v)=g_{\mu \nu} v^{\mu} v^{\nu}, \\
v^{\alpha} \frac{\partial}{\partial v^{\alpha}} g_{\mu \nu}=v^{\alpha} \frac{\partial}{\partial v^{\mu}} g_{\alpha \nu}=0,
\end{gathered}
$$

and

$$
\frac{\partial}{\partial v^{\alpha}} g_{\mu \nu}=\frac{\partial}{\partial v^{\mu}} g_{\alpha \nu} .
$$

In considering Finslerian spacetime and associated embedded Finslerian fields, it is useful to consider the tangent bundle of spacetime with spacetime in the base manifold and four-velocity space in the fiber. Using the homogeneity relations, Eqs.(4)-(9), then the connection and Riemann curvature scalar of the spacetime tangent bundle [6] can be significantly reduced for the case of a Finsler-spacetime base manifold [4], [7]. However, the special homogeneity requirements may not hold physically in general, but may only hold in certain special spacetime models.

A physical basis for the differential geometric structure of the spacetime tangent bundle is provided by the universal upper limit $a_{0}$ on proper acceleration $a$ relative to the vacuum [8]-[11]. If the proper acceleration were sufficiently large, then, because of vacuum radiation in an accelerated frame (in which particles are produced with average energy proportional to the proper acceleration), particles would be produced with mass such that their Schwarzschild radius exceeds their extent (Compton wavelength). Copious production of black-hole anti-black-hole pairs would ensue, accompanied by breakdown of the classical spacetime structure, and the very concept of acceleration would loose any meaning because of the resulting complex topology. Explicitly, the maximal proper acceleration, $a_{0}$, is given by $[8]$

$$
a_{0}=2 \pi \alpha\left(\frac{c^{7}}{\hbar G}\right)^{1 / 2},
$$

where $\alpha$ is a number of order unity, $c$ is the speed of light, $\hbar$ is Planck's constant divided by $2 \pi$, and $G$ is the universal gravitational constant. This is the 
maximum possible proper acceleration relative to the vacuum and is taken to be universal. Hence for any proper acceleration $a$, one requires

$$
a^{2} \leq a_{0}^{2}
$$

But, according to the differential geometry of spacetime, the proper acceleration, $a$, along a worldline in curved spacetime is given by

$$
a^{2}=-c^{4} g_{\mu \nu} \frac{D v^{\mu}}{d s} \frac{D v^{\nu}}{d s},
$$

where the four-velocity $v^{\mu}$ is given by

$$
v^{\mu}=\frac{d x^{\mu}}{d s}
$$

and $\frac{D v^{\mu}}{d s}$ denotes the covariant derivative of the four-velocity with respect to the interval along the worldline, namely,

$$
\frac{D v^{\mu}}{d s}=\frac{d v^{\mu}}{d s}+\Gamma_{\alpha \beta}^{\mu} v^{\alpha} v^{\beta},
$$

in which $\Gamma_{\alpha \beta}^{\mu}$ is the spacetime affine connection, and

$$
d s^{2} \equiv g_{\mu \nu} d x^{\mu} d x^{\nu}
$$

is the line element of spacetime. Then substituting Eqs.(12) and (14) in Eq.(11), one obtains

$$
-c^{4} g_{\mu \nu}\left(\frac{d v^{\mu}}{d s}+\Gamma_{\alpha \beta}^{\mu} v^{\alpha} v^{\beta}\right)\left(\frac{d v^{\nu}}{d s}+\Gamma_{\lambda \delta}^{\nu} v^{\lambda} v^{\delta}\right) \leq a_{0}^{2} .
$$

Next substituting Eqs.(13) and (15) in Eq.(16), one obtains [10]

$$
d \sigma^{2} \equiv g_{\mu v} d x^{\mu} d x^{\nu}+\rho_{0}^{2} g_{\mu v}\left(d v^{\mu}+\Gamma_{\alpha \beta}^{\mu} v^{\alpha} d x^{\beta}\right)\left(d v^{\nu}+\Gamma_{\lambda \delta}^{\nu} v^{\lambda} d x^{\delta}\right) \geq 0,
$$

where

$$
\rho_{0}=\frac{c^{2}}{a_{0}},
$$

is the minimum radius of curvature of worldlines. Equation (17) defines the eight-dimensional quadratic form $d \sigma^{2}$, which is nonnegative along the worldline. The inequality, Eq. (17), simply expresses the fact that the proper acceleration can never exceed the maximal proper acceleration. By analogy with the construction of the spacetime line element of general relativity from the limiting speed of light, it is natural to take $d \sigma^{2}$ to be the line element in the tangent bundle of spacetime, in which the spacetime coordinates $x^{\mu}$ are the coordinates in the spacetime base manifold, and the four-velocity coordinates $\rho_{0} v^{\mu}$ (modulo a factor of $\rho_{0}$ ) are the tangent space coordinates. 
The bundle line element, Eq.(17), can be rewritten as follows [10], [1]:

$$
d \sigma^{2} \equiv G_{M N} d x^{M} d x^{N}, \quad\{M, N=0,2, \ldots, 7\},
$$

where the bundle coordinates are

$$
\left\{x^{M}\right\} \equiv\left\{x^{\mu}, \rho_{0} v^{\mu}\right\}, \quad\{M=0,2, \ldots, 7 ; \mu=0,1,2,3\},
$$

and the metric of the tangent bundle of spacetime is

$$
G_{M N}=\left[\begin{array}{cc}
g_{\mu \nu}+g_{\alpha \beta} A^{\alpha}{ }_{\mu} A^{\beta}{ }_{\nu} & A_{n \mu} \\
A_{m \nu} & g_{m n}
\end{array}\right],
$$

in which

$$
A_{\nu}^{\mu}=\rho_{0} v^{\lambda} \Gamma_{\lambda \nu}^{\mu}
$$

The bundle metric $G_{M N}$, given by Eq.(21), has a structure similar to that of an eight-dimensional Kaluza-Klein gauge theory in which the higher dimensions are in four-velocity space, and $A^{\mu}{ }_{\nu}$ is the gauge potential. Eqs.( 19)-(22) served as the starting point for investigating possible implications of a limiting proper acceleration for the differential geometric structure of the tangent bundle of spacetime [1]-[4], [6], [8]-[24]. Possible forms for the bundle connection, curvature, and action were explored, including those based on Riemannian and Finsler spacetimes, and also Kähler and complex spacetime tangent bundles. Among the many differential geometric invariants of the spacetime tangent bundle, important for the present discussions is the Laplace-Beltrami operator:

$$
\mathcal{L}=G^{-1 / 2} \frac{\partial}{\partial x^{M}}\left(G^{1 / 2} G^{M N} \frac{\partial}{\partial x^{N}}\right) .
$$

This is the invariant generalization of the wave operator, or d'Alembertian, of field theory. A simple invariant field equation for a Finslerian scalar field $\phi(x, v)$ is then given by $[20]$

$$
\mathcal{L} \phi(x, v)=0 .
$$

\section{FINSLERIAN SCALAR QUANTUM FIELD}

When the spacetime is Minkowskian, the ordinary inhomogeneous Lorentz group (or Poincaré group) is a subgroup of the invariance group of the spacetime tangent bundle [12], [20]. It is of interest to examine quantum field solutions to Eq.(24) for this simple case, in order to establish connections between the Finslerian framework and canonical relativistic quantum field theory. To this end, consider a simple case in which the spacetime is completely flat. In particular, take the spacetime metric to be Minkowskian. For this case, it was argued in earlier work that the scalar field satisfying Eq.(24) is given by [20], [25], [26]:

$$
\begin{aligned}
\phi(x, v)=\int & \frac{d^{3} \mathbf{p}}{(2 \pi \hbar)^{3 / 2}\left(2 p^{0} N\right)^{1 / 2}}\left[e^{-i p x / \hbar} e^{-\rho_{0} p v / \hbar} \theta_{1}\left(\rho_{0} p v / \hbar\right) a(\mathbf{p})\right. \\
& \left.+e^{i p x / \hbar} e^{\rho_{0} p v / \hbar} \theta_{1}\left(-\rho_{0} p v / \hbar\right) a^{\dagger}(\mathbf{p})\right],
\end{aligned}
$$


where $p$ denotes the four-momentum $p^{\mu}=\left\{p^{0}, p^{1}, p^{2}, p^{3}\right\}$ of a particle excitation of the scalar field, $a^{\dagger}(\mathbf{p})$ and $a(\mathbf{p})$ are the particle creation and annihilation operators satisfying the commutation relations

$$
\left[a(\mathbf{p}), a^{\dagger}\left(\mathbf{p}^{\prime}\right)\right]=\delta^{3}\left(\mathbf{p}-\mathbf{p}^{\prime}\right), \quad\left[a(\mathbf{p}), a\left(\mathbf{p}^{\prime}\right)\right]=0, \quad\left[a^{\dagger}(\mathbf{p}), a^{\dagger}\left(\mathbf{p}^{\prime}\right)\right]=0,
$$

$\delta^{3}(\mathbf{z})$ is the three-dimensional Dirac delta function, and $\theta_{1}(z)$ is the Heaviside function [28],

$$
\theta_{1}(z)=\left\{\begin{array}{l}
1, \quad z \geq 0 \\
0, \quad z<0
\end{array} .\right.
$$

Also in Eq.(25), $N$ is a normalization factor such that the field operator acting on the vacuum state, namely $\langle 0| \phi(x, v)$, is the state of the field corresponding to a single-particle excitation appearing at some point $(x, v)$ in the spacetime tangent bundle, namely [25], [26],

$$
N=\frac{\rho_{0} V}{16 \pi^{2} \hbar^{2} m^{2} c^{2}} K_{1}\left(2 \rho_{0} m c / \hbar\right),
$$

where $V$ denotes the volume of space, $m$ is the mass of the scalar particle excitation of the quantum field, and $K_{1}(z)$ is the modified Bessel function of the third kind of order one.

It can be shown that both the positive and negative frequency terms in Eq.(25) are proportional to [20], [9]:

$$
e^{-\rho_{0}|p v| / \hbar}=\exp \left\{-\frac{1}{2 \pi \alpha}\left(\frac{G}{\hbar c}\right)^{1 / 2} \gamma m\left(\left[1+\left(\frac{|\mathbf{p}|}{m c}\right)^{2}\right]^{1 / 2}-\frac{\mathbf{p} \cdot d \mathbf{x} / d t}{m c^{2}}\right)\right\},
$$

where $d \mathbf{x} / d t$ is the ordinary velocity of the observer, and

$$
\gamma=\left[1-\left(\frac{|d \mathbf{x} / d t|}{c}\right)^{2}\right]^{-1 / 2} .
$$

As can be seen from Eq.(29), there occurs an intrinsic Planck-scale regularization of the quantum field, with an exponential energy cutoff beyond the Planck energy.

It is important to note that for an observer with ordinary velocity

$$
\dot{\mathbf{x}} \equiv d \mathbf{x} / d t
$$

in Minkowski spacetime, one has

$$
d s= \pm \sqrt{\left(d x^{0}\right)^{2}-|d \mathbf{x}|^{2}}= \pm \frac{d x^{0}}{\gamma},
$$

and therefore

$$
v^{\mu} \equiv \frac{d x^{\mu}}{d s}= \pm\{\gamma, \gamma \dot{\mathbf{x}} / c\}
$$


Thus the ordinary velocity $\dot{\mathbf{x}}$ corresponds to both future and past directed four velocity. This is essential in order that both the positive-frequency particle modes and the negative-frequency antiparticle modes in Eq.(25) and in the following have nonvanishing support. One is reminded of the Stückelberg-Feynman idea that antiparticles can be represented as particles with proper time reversed relative to true time [21], [27].

\section{HAMILTONIAN OF SCALAR QUANTUM FIELD}

On the basis of microcausality, it was argued in earlier work [21] that it is logical to define the generalized adjoint $(\phi(x, v))^{\dagger}$ of the quantum field $\phi(x, v)$ by

$$
(\phi(x, v))^{\dagger}=\phi^{\dagger}(x,-v),
$$

in which the ordinary adjoint is taken, but also the sign of the four-velocity $v$ is changed. This is connected, through charge-conjugation, with the fact that the positive-frequency particle component of the field in Eq.(25) has nonvanishing support for positive $v^{0}$ (or equivalently, positive $p v$ ), and the negative- frequency anti-particle component has nonvanishing support for negative $v^{0}$ (equivalently, negative $p v$ ) [21]. In order that the Hamiltonian be Hermitian (in terms of the generalized adjoint), that it reduce to the canonical form in Fock space, and that the observer's four-velocity lie on the four-velocity shell $\left(v^{2}=1\right)$, it is natural to define the Hamiltonian for the scalar quantum field as follows [25], [26]:

$$
H=\int \rho_{0}^{4} d^{4} v \delta\left(\rho_{0}^{2} v^{2}-\rho_{0}^{2}\right) d^{3} \mathbf{x} T_{00}(\mathbf{x}, v),
$$

in which the bundle energy density $T_{00}(\mathbf{x}, v)$ at point $(x, v)$ in the bundle is integrated over the entire spacetime tangent bundle with large spatial volume $V, \boldsymbol{\delta}(z)$ is the one-dimensional Dirac delta function, and the bundle energy density is given by

$$
\begin{gathered}
T_{00}(\mathbf{x}, v)=\frac{V}{32 \pi^{3} \hbar m}\left[\frac{\partial}{\partial x^{0}} \phi(\mathbf{x},-v) \frac{\partial}{\partial x^{0}} \phi(\mathbf{x}, v)+\nabla_{\mathbf{x}} \phi(\mathbf{x},-v) \cdot \nabla_{\mathbf{x}} \phi(\mathbf{x}, v)\right. \\
\left.+\frac{1}{\rho_{0}^{2}} \frac{\partial}{\partial v^{\mu}} \phi(\mathbf{x},-v) \frac{\partial}{\partial v_{\mu}} \phi(\mathbf{x}, v)\right] .
\end{gathered}
$$

To see that the Hamiltonian is Hermitian, one first notes that

$$
H^{\dagger}=\int \rho_{0}^{4} d^{4} v \delta\left(\rho_{0}^{2} v^{2}-\rho_{0}^{2}\right) d^{3} \mathbf{x} T_{00}(\mathbf{x}, v)^{\dagger} .
$$

Next, taking the generalized adjoint of $T_{00}(\mathbf{x}, v)$ and using Eq.(34), one obtains

$$
\begin{gathered}
T_{00}(\mathbf{x}, v)^{\dagger}=\frac{V}{32 \pi^{3} \hbar m}\left[\frac{\partial}{\partial x^{0}} \phi^{\dagger}(\mathbf{x},-v) \frac{\partial}{\partial x^{0}} \phi^{\dagger}(\mathbf{x}, v)+\nabla_{\mathbf{x}} \phi^{\dagger}(\mathbf{x},-v) \cdot \nabla_{\mathbf{x}} \phi^{\dagger}(\mathbf{x}, v)\right. \\
\left.+\frac{1}{\rho_{0}^{2}} \frac{\partial}{\partial v^{\mu}} \phi^{\dagger}(\mathbf{x},-v) \frac{\partial}{\partial v_{\mu}} \phi^{\dagger}(\mathbf{x}, v)\right] .
\end{gathered}
$$


Equivalently, using Eq.(25), Eq.(38) becomes

$$
\begin{aligned}
T_{00}(\mathbf{x}, v)^{\dagger}=\frac{V}{32 \pi^{3} \hbar m}[ & \frac{\partial}{\partial x^{0}} \phi(\mathbf{x}, v) \frac{\partial}{\partial x^{0}} \phi(\mathbf{x},-v)+\nabla_{\mathbf{x}} \phi(\mathbf{x}, v) \cdot \nabla_{\mathbf{x}} \phi(\mathbf{x},-v) \\
& \left.+\frac{1}{\rho_{0}^{2}} \frac{\partial}{\partial v^{\mu}} \phi(\mathbf{x}, v) \frac{\partial}{\partial v_{\mu}} \phi(\mathbf{x},-v)\right],
\end{aligned}
$$

or comparing Eqs.(39) and (36), one obtains

$$
T_{00}(\mathbf{x}, v)^{\dagger}=T_{00}(\mathbf{x},-v) .
$$

Next substituting Eq.(40) in Eq.(37), one has

$$
H^{\dagger}=\int \rho_{0}^{4} d^{4} v \delta\left(\rho_{0}^{2} v^{2}-\rho_{0}^{2}\right) d^{3} \mathbf{x} T_{00}(\mathbf{x},-v),
$$

and replacing the dummy variable of integration $v$ by $-v$, Eq.(41) becomes

$$
H^{\dagger}=\int \rho_{0}^{4} d^{4} v \delta\left(\rho_{0}^{2} v^{2}-\rho_{0}^{2}\right) d^{3} \mathbf{x} T_{00}(\mathbf{x}, v) .
$$

Finally, substituting Eq.(35) in Eq.(42), one concludes that

$$
H^{\dagger}=H \text {. }
$$

Thus $H^{\dagger}$ is in fact Hermitian.

Next, using Eqs.(25), (27) and (36) in Eq.(35), integrating over space, and substituting the integral [25], [29]

$$
\int \frac{d^{3} \mathbf{v}}{2 v^{0}} e^{-2 \rho_{0} p v / \hbar}=\frac{\pi \hbar}{\rho_{0} m c} K_{1}\left(2 \rho_{0} m c / \hbar\right),
$$

one obtains the canonical expression for the Hamiltonian operator for a scalar quantum field in Fock space [25]:

$$
H=\frac{1}{2} c \int d^{3} \mathbf{p} p^{0}\left[a^{\dagger}(\mathbf{p}) a(\mathbf{p})+a(\mathbf{p}) a^{\dagger}(\mathbf{p})\right],
$$

in which

$$
p^{0}=\left(m^{2} c^{2}+|\mathbf{p}|^{2}\right)^{1 / 2} .
$$

For consistency, it is also well to verify that the Heisenberg equation of motion for the field $\phi$ is satisfied, namely [30],

$$
[H, \phi]=-i \hbar \frac{\partial}{\partial t} \phi .
$$

To see that Eq.(47) holds, one uses Eqs.(45) and (25) to evaluate the commutator $[H, \phi]$. Thus one has

$$
\begin{aligned}
{[H, \phi]=} & \frac{1}{2} c \int d^{3} \mathbf{p}^{\prime} p^{0 \prime}\left[\left(a^{\dagger}\left(\mathbf{p}^{\prime}\right) a\left(\mathbf{p}^{\prime}\right)+a\left(\mathbf{p}^{\prime}\right) a^{\dagger}\left(\mathbf{p}^{\prime}\right)\right), \int \frac{d^{3} \mathbf{p}}{(2 \pi \hbar)^{3 / 2}\left(2 p^{0} N\right)^{1 / 2}}\right. \\
& \left\{e^{-i p x / \hbar} e^{-\rho_{0} p v / \hbar} \theta_{1}\left(\rho_{0} p v / \hbar\right) a(\mathbf{p})\right. \\
& \left.\left.+e^{i p x / \hbar} e^{\rho_{0} p v / \hbar} \theta_{1}\left(-\rho_{0} p v / \hbar\right) a^{\dagger}(\mathbf{p})\right\}\right],
\end{aligned}
$$


or equivalently,

$$
\begin{aligned}
{[H, \phi]=} & \frac{1}{2} c \int \frac{d^{3} \mathbf{p}^{\prime} p^{0 \prime} d^{3} \mathbf{p}}{(2 \pi \hbar)^{3 / 2}\left(2 p^{0} N\right)^{1 / 2}}\left\{e^{-i p x / \hbar} e^{-\rho_{0} p v / \hbar} \theta_{1}\left(\rho_{0} p v / \hbar\right)\right. \\
& \left(\left[a^{\dagger}\left(\mathbf{p}^{\prime}\right) a\left(\mathbf{p}^{\prime}\right), a(\mathbf{p})\right]+\left[a\left(\mathbf{p}^{\prime}\right) a^{\dagger}\left(\mathbf{p}^{\prime}\right), a(\mathbf{p})\right]\right) \\
& +e^{i p x / \hbar} e^{\rho_{0} p v / \hbar} \theta_{1}\left(-\rho_{0} p v / \hbar\right) \\
& \left.\left(\left[a^{\dagger}\left(\mathbf{p}^{\prime}\right) a\left(\mathbf{p}^{\prime}\right), a^{\dagger}(\mathbf{p})\right]+\left[a\left(\mathbf{p}^{\prime}\right) a^{\dagger}\left(\mathbf{p}^{\prime}\right), a^{\dagger}(\mathbf{p})\right]\right)\right\}
\end{aligned}
$$

Then substituting Eqs.(26) in Eq.(49), one obtains

$$
\begin{aligned}
{[H, \phi]=} & -c \int \frac{d^{3} \mathbf{p} p^{0}}{(2 \pi \hbar)^{3 / 2}\left(2 p^{0} N\right)^{1 / 2}}\left\{e^{-i p x / \hbar} e^{-\rho_{0} p v / \hbar} \theta_{1}\left(\rho_{0} p v / \hbar\right) a(\mathbf{p})\right. \\
& \left.-e^{i p x / \hbar} e^{\rho_{0} p v / \hbar} \theta_{1}\left(-\rho_{0} p v / \hbar\right) a^{\dagger}(\mathbf{p})\right\} .
\end{aligned}
$$

Finally, substituting Eq.(25) in Eq.(50), one obtains the Heisenberg equation of motion for the field, Eq.(47).

\section{VACUUM ENERGY DENSITY}

Using Eqs.(35) and (36), and denoting the vacuum state by $|0\rangle$, it follows that the vacuum energy in the bundle, for spatial volume $V$, is given by [25]:

$$
\langle 0|H| 0\rangle=\frac{1}{2} c V \int \frac{d^{3} \mathbf{p}}{(2 \pi \hbar)^{3}}\left(m^{2} c^{2}+|\mathbf{p}|^{2}\right)^{1 / 2},
$$

which is the canonical divergent result [30]. However, a particular observer has four-velocity $v$, and his worldline at any time is confined to the neighborhood of $v$ in the fiber (See Eq.(33)). The vacuum energy which he observes is therefore given by [25]

$$
\langle 0|\Delta H| 0\rangle=\rho_{0}^{3} \Delta^{3} \mathbf{v}\left\langle 0\left|\int \rho_{0} d v^{0} \delta\left(\rho_{0}^{2} v^{2}-\rho_{0}^{2}\right) d^{3} \mathbf{x} T_{00}(\mathbf{x}, v)\right| 0\right\rangle,
$$

in which $\Delta^{3} \mathbf{v}$ is defined by

$$
\Delta^{3} \mathbf{v} \equiv v^{0} \int_{\mathbf{v}-\delta \mathbf{v} / 2}^{\mathbf{v}+\delta \mathbf{v} / 2} \frac{d^{3} \mathbf{v}^{\prime}}{v^{0 \prime}} \underset{\delta \mathbf{v} \ll \mathbf{v}}{\longrightarrow} \delta^{3} \mathbf{v},
$$

where $\delta \mathbf{v}$ is the spread in the spatial components of the four-velocity of the observer, and because of the Dirac delta function in Eq.(52),

$$
v^{0 \prime}=\left(1+\left|\mathbf{v}^{\prime}\right|^{2}\right)^{1 / 2}, \quad v^{0}=\left(1+|\mathbf{v}|^{2}\right)^{1 / 2} .
$$


One notes that in Eq.(52), one can use the well known identity:

$$
\begin{aligned}
& \delta\left(\rho_{0}^{2} v^{2}-\rho_{0}^{2}\right) \\
= & \frac{1}{\rho_{0}^{2}} \delta\left(\left\{v^{0}+\left[1+|\mathbf{v}|^{2}\right]^{1 / 2}\right\}\left\{v^{0}-\left[1+|\mathbf{v}|^{2}\right]^{1 / 2}\right\}\right) \\
= & \frac{1}{2 \rho_{0}^{2}\left[1+|\mathbf{v}|^{2}\right]^{1 / 2}}\left[\delta\left(v^{0}-\left[1+|\mathbf{v}|^{2}\right]^{1 / 2}\right)+\delta\left(v^{0}+\left[1+|\mathbf{v}|^{2}\right]^{1 / 2}\right)\right] .
\end{aligned}
$$

Equation (53), defining the spread, is true because the expression following $\Delta^{3} \mathbf{v}$ in Eq. (52) turns out to be proportional to $\left(1 / v^{0}\right)=\left(1+|\mathbf{v}|^{2}\right)^{-1 / 2}$, with no other dependence on the spatial component $\mathbf{v}$ of four-velocity (See Eqs.(54) and (62)-(67)).

Next, denoting the three respective contributions to Eq.(52) of the three terms of Eq.(36) by $\left\langle 0\left|\Delta H_{1}\right| 0\right\rangle,\left\langle 0\left|\Delta H_{2}\right| 0\right\rangle$, and $\left\langle 0\left|\Delta H_{3}\right| 0\right\rangle$, respectively, then

$$
\langle 0|\Delta H| 0\rangle=\left\langle 0\left|\Delta H_{1}\right| 0\right\rangle+\left\langle 0\left|\Delta H_{2}\right| 0\right\rangle+\left\langle 0\left|\Delta H_{3}\right| 0\right\rangle .
$$

Then using Eqs.(36), (52), and (56), one has

$$
\begin{aligned}
& \left\langle 0\left|\Delta H_{1}\right| 0\right\rangle \\
= & \frac{V}{32 \pi^{3} \hbar m} \rho_{0}^{4} \Delta^{3} \mathbf{v}\left\langle 0\left|\int d v^{0} \delta\left(\rho_{0}^{2} v^{2}-\rho_{0}^{2}\right) d^{3} \mathbf{x} \frac{\partial}{\partial x^{0}} \phi(\mathbf{x},-v) \frac{\partial}{\partial x^{0}} \phi(\mathbf{x}, v)\right| 0\right\rangle,
\end{aligned}
$$

and substituting Eqs.(25) and (55) in Eq.(57), one obtains

$$
\begin{aligned}
& \left\langle 0\left|\Delta H_{1}\right| 0\right\rangle \\
= & \frac{V}{32 \pi^{3} \hbar m} \rho_{0}^{2} \Delta^{3} \mathbf{v} \int \frac{d v^{0}}{2\left[1+|\mathbf{v}|^{2}\right]^{1 / 2}}\left[\delta\left(v^{0}-\left[1+|\mathbf{v}|^{2}\right]^{1 / 2}\right)\right. \\
& \left.+\delta\left(v^{0}+\left[1+|\mathbf{v}|^{2}\right]^{1 / 2}\right)\right] d^{3} \mathbf{x} \frac{d^{3} \mathbf{p} d^{3} \mathbf{p}^{\prime}}{(2 \pi \hbar)^{3} 2 N\left(p^{0} p^{0 \prime}\right)^{1 / 2}} \\
& \langle 0|\left\{-i \frac{p^{0}}{\hbar} e^{-i p x / \hbar} e^{\rho_{0} p v / \hbar} \theta_{1}\left(-v^{0}\right) a(\mathbf{p})+i \frac{p^{0}}{\hbar} e^{i p x / \hbar} e^{-\rho_{0} p v / \hbar} \theta_{1}\left(v^{0}\right) a^{\dagger}(\mathbf{p})\right\} \\
& \left\{-i \frac{p^{0 \prime}}{\hbar} e^{-i p^{\prime} x / \hbar} e^{-\rho_{0} p^{\prime} v / \hbar} \theta_{1}\left(v^{0}\right) a\left(\mathbf{p}^{\prime}\right)+i \frac{p^{0 \prime}}{\hbar} e^{i p^{\prime} x / \hbar} e^{\rho_{0} p^{\prime} v / \hbar} \theta_{1}\left(-v^{0}\right) a^{\dagger}\left(\mathbf{p}^{\prime}\right)\right\}|0\rangle .
\end{aligned}
$$

Here $\theta_{1}\left( \pm \rho_{0} p v / \hbar\right)$ has been replaced by $\theta_{1}\left( \pm v^{0}\right)$, since it can be shown that $p v>m c$ when $v^{0}=\gamma>0$, and $p v<m c$ when $v^{0}=-\gamma<0$ [20], [21]. Then, according to Eqs.(27), (30) and (54), one has

$$
\theta_{1}\left(v^{0}\right) \theta_{1}\left(-v^{0}\right)=0, \quad\left[\theta_{1}\left(v^{0}\right)\right]^{2}=\theta_{1}\left(v^{0}\right)
$$


Next using Eq.(59) in Eq.(58), one obtains

$$
\begin{aligned}
& \left\langle 0\left|\Delta H_{1}\right| 0\right\rangle \\
= & \frac{V}{32 \pi^{3} \hbar m} \rho_{0}^{2} \Delta^{3} \mathbf{v} \int \frac{d v^{0}}{2\left[1+|\mathbf{v}|^{2}\right]^{1 / 2}}\left[\delta\left(v^{0}-\left[1+|\mathbf{v}|^{2}\right]^{1 / 2}\right)\right. \\
& \left.+\delta\left(v^{0}+\left[1+|\mathbf{v}|^{2}\right]^{1 / 2}\right)\right] d^{3} \mathbf{x} \frac{d^{3} \mathbf{p} d^{3} \mathbf{p}^{\prime}}{(2 \pi \hbar)^{3} 2 N\left(p^{0} p^{0 \prime}\right)^{1 / 2}} \\
& \left\{\frac{p^{0} p^{0 \prime}}{\hbar^{2}} e^{-i\left(p-p^{\prime}\right) x / \hbar} e^{\rho_{0}\left(p+p^{\prime}\right) v / \hbar} \theta_{1}\left(-v^{0}\right)\left\langle 0\left|a(\mathbf{p}) a^{\dagger}\left(\mathbf{p}^{\prime}\right)\right| 0\right\rangle\right. \\
& \left.+\frac{p^{0} p^{0 \prime}}{\hbar^{2}} e^{i\left(p-p^{\prime}\right) x / \hbar} e^{-\rho_{0}\left(p+p^{\prime}\right) v / \hbar} \theta_{1}\left(v^{0}\right)\left\langle 0\left|a^{\dagger}(\mathbf{p}) a\left(\mathbf{p}^{\prime}\right)\right| 0\right\rangle\right\} .
\end{aligned}
$$

One also has

$$
a(\mathbf{p})|0\rangle=0, \quad\left\langle 0\left|a^{\dagger}(\mathbf{p})=0, \quad\right| \mathbf{p}\right\rangle=a^{\dagger}(\mathbf{p})|0\rangle, \quad\left\langle\mathbf{p} \mid \mathbf{p}^{\prime}\right\rangle=\delta^{3}\left(\mathbf{p}-\mathbf{p}^{\prime}\right) .
$$

Therefore using Eqs.(61) in Eq.(60), one obtains

$$
\begin{aligned}
& \left\langle 0\left|\Delta H_{1}\right| 0\right\rangle \\
= & \frac{V}{32 \pi^{3} \hbar m} \rho_{0}^{2} \Delta^{3} \mathbf{v} \int \frac{d v^{0}}{2\left[1+|\mathbf{v}|^{2}\right]^{1 / 2}}\left[\delta\left(v^{0}-\left[1+|\mathbf{v}|^{2}\right]^{1 / 2}\right)\right. \\
& \left.+\delta\left(v^{0}+\left[1+|\mathbf{v}|^{2}\right]^{1 / 2}\right)\right] d^{3} \mathbf{x} \frac{d^{3} \mathbf{p} d^{3} \mathbf{p}^{\prime}}{(2 \pi \hbar)^{3} 2 N\left(p^{0} p^{0 \prime}\right)^{1 / 2}} \\
& \frac{p^{0} p^{0 \prime}}{\hbar^{2}} e^{-i\left(p-p^{\prime}\right) x / \hbar} e^{\rho_{0}\left(p+p^{\prime}\right) v / \hbar} \theta_{1}\left(-v^{0}\right) \delta^{3}\left(\mathbf{p}-\mathbf{p}^{\prime}\right),
\end{aligned}
$$

or equivalently,

$$
\left\langle 0\left|\Delta H_{1}\right| 0\right\rangle=\frac{V^{2}}{32 \pi^{3} \hbar^{3} m} \frac{1}{(2 \pi \hbar)^{3} 4 N} \frac{\rho_{0}^{2} \Delta^{3} \mathbf{v}}{v^{0}} \int \frac{d^{3} \mathbf{p}}{p^{0}} p^{02} e^{-2 \rho_{0} p v / \hbar}
$$

Analogously, corresponding to the contributions to Eq.(56) of the two remaining terms of Eq.(38), containing derivatives with respect to space and four-velocity, respectively, one obtains

$$
\left\langle 0\left|\Delta H_{2}\right| 0\right\rangle=\frac{V^{2}}{32 \pi^{3} \hbar^{3} m} \frac{1}{(2 \pi \hbar)^{3} 4 N} \frac{\rho_{0}^{2} \Delta^{3} \mathbf{v}}{v^{0}} \int \frac{d^{3} \mathbf{p}}{p^{0}}|\mathbf{p}|^{2} e^{-2 \rho_{0} p v / \hbar},
$$

and

$$
\left\langle 0\left|\Delta H_{3}\right| 0\right\rangle=\frac{V^{2}}{32 \pi^{3} \hbar^{3} m} \frac{1}{(2 \pi \hbar)^{3} 4 N} \frac{\rho_{0}^{2} \Delta^{3} \mathbf{v}}{v^{0}} \int \frac{d^{3} \mathbf{p}}{p^{0}} m^{2} c^{2} e^{-2 \rho_{0} p v / \hbar} .
$$

Then substituting Eqs.(63)-(65) in Eq.(56), noting that

$$
p^{02}=|\mathbf{p}|^{2}+m^{2} c^{2}
$$


evaluating the following integral [26], [29],

$$
\int d^{3} \mathbf{p} p^{0} e^{-2 \rho_{0} p v / \hbar}=\frac{\pi \hbar^{2} m^{2} c^{2}}{\rho_{0}^{2}} K_{2}\left(2 \rho_{0} m c / \hbar\right),
$$

where $K_{2}(z)$ is the modified Bessel function of the third kind of order 2, substituting Eq.(28), and dividing by the spatial volume $V$, one finally obtains the vacuum energy density seen by an observer at $(x, v)$ in the spacetime tangent bundle:

$$
\frac{\langle 0|\Delta H| 0\rangle}{V}=\frac{m^{3} c^{4}}{32 \pi^{3} \hbar^{2} \rho_{0}} \frac{K_{2}\left(2 \rho_{0} m c / \hbar\right)}{K_{1}\left(2 \rho_{0} m c / \hbar\right)} \frac{\Delta^{3} \mathbf{v}}{v^{0}} .
$$

Thus any one observer sees a finite vacuum energy density given by Eq.(68). One notes that for sufficiently small spread in the spatial part of the observer's fourvelocity (Eq.(53)), the observed vacuum energy density may be near vanishing. This may be consistent with a near vanishing cosmological constant.

\section{References}

[1] Brandt, H. E. (1996) Finslerian Spacetime, Contemporary Math., 196, 273287.

[2] Brandt, H. E. (1999) Finslerian Fields in the Spacetime Tangent Bundle, Chaos, Solitons Fractals, 10, 267-282.

[3] Brandt, H. E. (2000) Finslerian Fields, in Finslerian Geometries: A Meeting of Minds, P. L. Antonelli, ed., Kluwer, Dordrecht, pp. 131-138.

[4] Brandt, H. E. (1992) Finsler-Spacetime Tangent Bundle, Found. Phys. Lett., 5, 221-248.

[5] H. Rund (1959) The Differential Geometry of Finsler Spaces, Berlin, Springer.

[6] Brandt, H. E. (1992) Riemann Curvature Scalar of Spacetime Tangent Bundle, Found. Phys. Lett., 5, 43-55.

[7] K. Yano and E. T. Davies (1963) On the Tangent Bundles of Finsler and Riemannian Manifolds, Rend. Circ. Mat. Palermo 12, 211-228.

[8] Brandt, H. E. (1983) Maximal Proper Acceleration Relative to the Vacuum, Lett. Nuovo Cim., 38, 522-524; (1984), 39, 192.

[9] Brandt, H. E. (2003) Quantum Vacuum Heuristics, J. Mod. Optics, 50, 2455-2463.

[10] Brandt, H. E. (1989) Maximal Proper Acceleration and the Structure of Spacetime, Found. Phys. Lett., 2, 39-58, 405. 
[11] Brandt, H. E. (1991) Structure of Spacetime Tangent Bundle, Found. Phys. Lett., 4, 523-536.

[12] Brandt, H. E. (1984) The Maximal Acceleration Group, in XIIIth International Colloquium on Group Theoretical Methods in Physics, W. W. Zachary, ed., World Scientific, Singapore, pp. 519-522.

[13] Brandt, H. E. (1991) Connections and Geodesics in the Spacetime Tangent Bundle, Found. Phys., 21, 1285-1295.

[14] Brandt, H. E. (1992) Differential Geometry of Spacetime Tangent Bundle, Int. J. Theor. Phys. 31, 575-580.

[15] Brandt, H. E. (1989) Kinetic Theory in Maximal-Acceleration Invariant Phase Space, Nucl. Phys. B (Proc. Suppl.), 6, 367-369.

[16] Brandt, H. E. (1992) Kähler Spacetime Tangent Bundle, Found. Phys. Lett., 5, 315-336.

[17] Brandt, H. E. (1993) Complex Spacetime Tangent Bundle, Found. Phys. Lett., 6, 245-255.

[18] Brandt, H. E. (1993) Spacetime Tangent Bundle with Torsion, Found. Phys. Lett., 6, 339-369.

[19] Brandt, H. E. (1994) Solution to Torsion Relations in Finsler-Spacetime Tangent Bundle, Found. Phys. Lett., 7, 297-301.

[20] Brandt, H. E. (1998) Quantum Fields in the Spacetime Tangent Bundle, Found. Phys. Lett., 11, 265-275.

[21] Brandt, H.E. (2000) Finslerian Quantum Fields and Microcausality, Found. Phys. Lett., 13, 307-328.

[22] Brandt, H. E. (2000) Causal Domain of Minkowski-Spacetime Tangent Bundle, Found. Phys. Lett., 13, 581-588.

[23] Brandt, H. E. (2000) Particle Geodesics and Spectra in the Spacetime Tangent Bundle, Rep. Math. Phys., 45, 389-405.

[24] Brandt, H. E. (2003) Lorentz-Invariant Quantum Fields in the Spacetime Tangent Bundle, Internat. J. Math. and Math. Sci., 2003, No. 24, 15291546 .

[25] Brandt, H. E. (2004) Intrinsic Gravitational Regularization of Quantum Fields, Found. Phys. Lett. 17, 295-300.

[26] Brandt, H. E. (2004) Finite Vacuum Energy Density in Quantum Field Theory, to appear in J. Mod. Optics (2004).

[27] Feynman, R. P. (1949) The Theory of Positrons, Phys. Rev. 76, 749-759. 
[28] Kanwal, R. P. (1983) Generalized Functions: Theory and Technique, Academic Press, New York, p.1.

[29] Oberhettinger, F. and Badii, L. (1973) Tables of Laplace Transforms, Springer, New York, p.94.

[30] Zee, A. (2003) Quantum Field Theory in a Nutshell, Princeton University Press, Princeton. 